\title{
INVESTIGAÇÕES GEOFÍSICAS EM ROCHAS GRANÍTICAS NO MUNICÍPIO DE ITU, SÃO PAULO - BRASIL
}

\author{
Jorge Luís Porsani, ' Francisco Yukio Hiodo² e Vagner Roberto Elis ${ }^{3}$ \\ Recebido em 15 jan., 2002 / Aceito em 4 nov., 2003 \\ Received Jan. 15, 2002 / Accepted Nov. 4, 2003
}

\begin{abstract}
Geophysical investigations was realized on the Granitic Suite of Itu, located near to Itu City, State of São Paulo - Brazil, with the objective to find fractures zones in granitic rocks and determine the depth of top of fresh rock, through of integration of GPR - Ground Penetrating Radar and Resistivity Methods. The geophysical profiles were realized on a same line for a comparative study between these two methodologies, inside of philosophy of integrated interpretation. One GPR profile of $80 \mathrm{~m}$ was acquired with 50, 100 and $200 \mathrm{MHz}$ antennas and two electric profiles with dipole of 2 and 10m. Analysis of geophysical profiles allowed to identify two strong dip reflectors: one about $10 \mathrm{~m}$ depth, interpreted as fractures filled with water and the other between 12 and $17 \mathrm{~m}$ depth that correspond to one resistive region dipping after of $15 \mathrm{~m}$ depth, interpreted as the top of granitic fresh rock. Besides, two anomalous regions were identified: one about $50 \mathrm{~m}$ and the other about 80m. In GPR profiles, these regions are characterized for one shadow zone, because the high attenuation of electromagnetic waves. In electric profiles, these regions correspond to the conductive regions that can be related with the presence of one sub-vertical fracture zone.
\end{abstract}

Keywords: GPR-Ground Penetrating Radar, Electrical Resistivity, Fractures Zones, Granite, Itu, São Paulo, Brazil.

\section{RESUMO}

Investigações geofísicas foram realizadas sobre a Suíte Granítica de Itu, localizada próxima à cidade de Itu, Estado de São Paulo-Brasil, com o objetivo de localizar zonas de fraturas nas rochas graníticas e determinar o topo da rocha sã, através da integração dos métodos GPR-Ground Penetrating Radar e Resistividade Elétrica. Os perfis geofísicos foram realizados sobre uma mesma linha para estudos comparativos entre estas duas metodologias, com a filosofia de interpretação integrada. Um perfil GPR de 80m foi adquirido com as antenas de 50, 100 e 200 MHz, além de dois perfis de caminhamento elétrico com dipólos de 2 e 10m. As análises dos perfis geofísicos permitiram identificar dois fortes refletores inclinados: um em torno de $10 \mathrm{~m}$ de profundidade, interpretado como fraturas preenchidas com água e o outro entre 12 e 17m de profundidade, que corresponde a uma região resistiva mergulhando depois $15 \mathrm{~m}$ de profundidade, interpretada como 0 topo da rocha granítica sã. Além disso, duas regiões anômalas foram identificadas: uma em torno de $50 \mathrm{~m}$ e a outra em torno de $80 \mathrm{~m}$. Nos perfis GPR, essas regiões são caracterizadas por uma região sem refletores, devido à elevada atenuação das ondas eletromagnéticas. Nos perfis elétricos, essas regiões correspondem às regiões condutivas que podem estar relacionadas com a presença de uma fratura subvertical.

Palavras-Chave: GPR-Ground Penetrating Radar, Resistividade elétrica, Granitos de Itu, fraturas.

\footnotetext{
IAG-USP. Departamento de Geofísica. Rua do Matão 1226. Cidade Universitária. São Paulo - SP. CEP 05508-090. Fone: 0xx11 3091 4734. Fax: 0xx11 3091 5034. Email: porsani@iag.usp.br

2 IAG-USP. Departamento de Geofísica. Rua do Matão 1226. Cidade Universitária. São Paulo - SP. CEP 05508-090. Fone: Oxx11 3091 4777. Fax: 0xx11 3091 5034. Email: francisc@iag.usp.br

3 IAG-USP. Departamento de Geofísica. Rua do Matão 1226. Cidade Universitária. São Paulo - SP. CEP 05508-090. Fone: Oxx11 3091 4734. Fax: 0xx11 3091 5034. Email: vagnelis@iag.usp.br
} 


\section{INTRODUÇÃOO}

Este trabalho tem como objetivo localizar zonas de fraturas em rochas graníticas e determinar a profundidade do topo da rocha sã, através da integração dos métodos GPR - Ground Penetrating Radar (PORSANI, 1999) e resistividade elétrica com arranjos dos eletrodos do tipo dipolodipolo (ELIS, 1999). Os perfis geofísicos foram realizados sobre uma mesma linha visando um estudo comparativo entre estas duas metodologias, dentro da filosofia de interpretação integrada. Os levantamentos de campo foram realizados sobre a Suíte Graníitica de Itu, situada numa área de proteção ambiental localizada na Fazenda da Serra, município de ltu, estado de São Paulo. Nessa região, as principais fontes de água de superfície estão bastante contaminadas e 0 abastecimento de água potável para a comunidade é realizado através da exploração de água subterrânea por meio de poços tubulares. Portanto, a aplicação de métodos geofísicos, visando a localização de zonas de fraturas em rochas cristalinas para a exploração de água subterrânea é de grande importância para a região.

\section{GEOLOGIA DA ÁREA DE ESTUDO}

A Suíte Granítica de Itu está localizada a aproximadamente $60 \mathrm{~km}$ da cidade de São Paulo, e abrange uma área de aproximadamente $400 \mathrm{~km} .{ }^{2}$ Segundo Pascholati (1990) a área compreende uma região de transição entre os terrenos Pré-Cambrianos do estado de São Paulo e a Bacia Sedimentar do Paraná.

As rochas encaixantes são principalmente gnáisses com intercalações de xistos, quartzitos, anfibolitos e granulitos pertencentes ao Complexo Piracaia. 0 limite ao Sul da Suíte coincide parcialmente com a Falha de Jundiuvira.

Devido à escassez de afloramentos e à cobertura por unidades geológicas mais recentes, as exposições dos contatos dos corpos granitóides com as rochas encaixantes são raras e quando observados podem ser tectônicos ou intrusivos.

Os contatos são caracterizados principalmente por zonas de cisalhamentos. As estruturas geológicas mais marcantes da Suíte Granítica de ltu são os lineamentos que correspondem às zonas de falhas curvadas, subparalelas ou convergentes à zona de falha de Jundiuvira. Essas zonas coincidem parcialmente com a orientação das principais drenagens da região, por exemplo, os rios Tietê, Pirá́ e da Fonte.

Os lineamentos retilíneos mais importantes que afetam os corpos granitóides apresentam orientação segundo os quadrantes NE e NW, onde são abundantes as direções preferenciais de N20-30E e N45-50W. Esses lineamentos representam geologicamente as zonas de falhas/ fraturas, que são importantes estruturas para a exploração de águas subterrâneas em rochas graníticas. A Figura 1 mostra um mapa de localização esquemático da área de estudos.

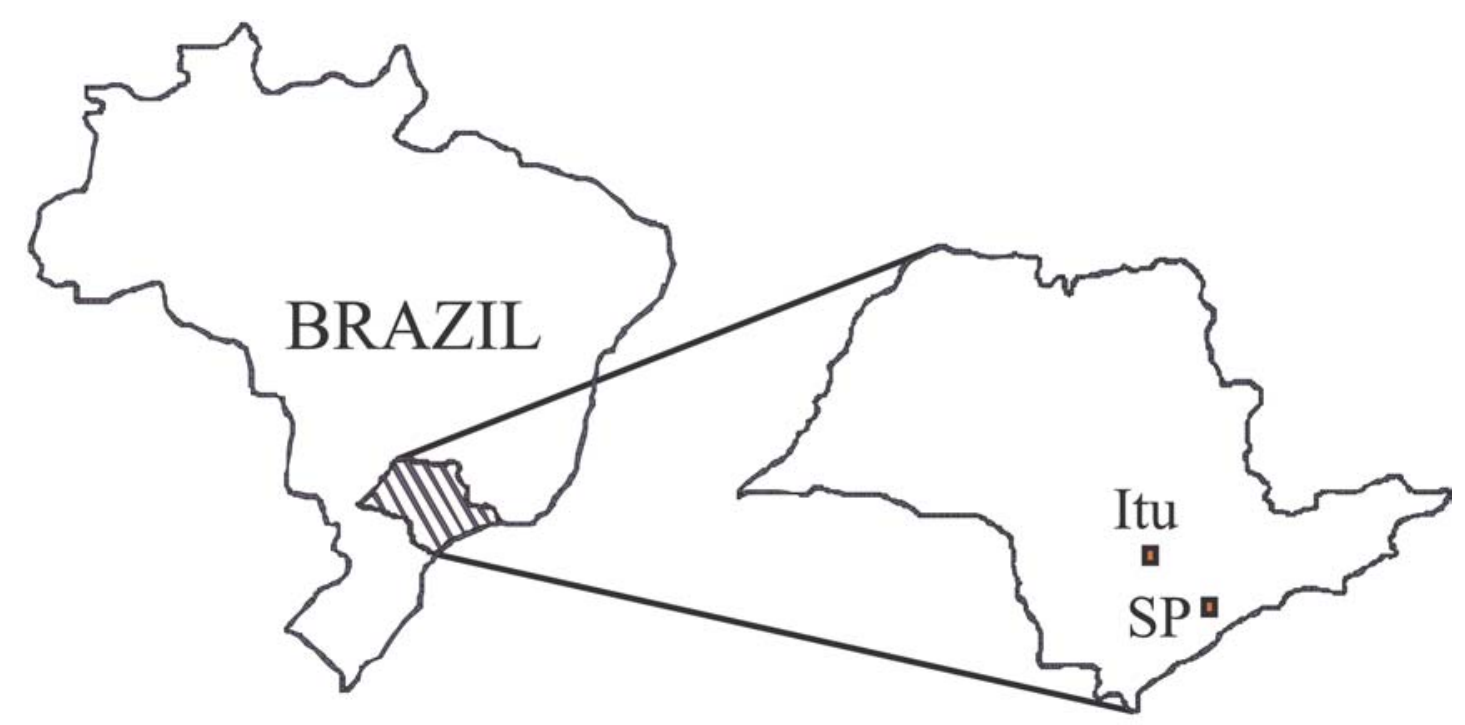

Figura 1 - Mapa de localização esquemático da área de estudos no Estado de São Paulo. Figure 1 - Location map of the studies area in São Paulo State. 


\section{AQUISIÇÃO E PROCESSAMENTO DOS DADOS GEOFÍSICOS}

0 posicionamento do perfil geofísico foi realizado com base nas facilidades logísticas, como a topografia, e na direção dos lineamentos geológicos encontrados na região. 0 local dos trabalhos é caracterizado por um relevo bastante acidentado. Dessa forma, procurou-se uma posição no terreno em que o perfil geofísico ficasse sub-horizontal, portanto, não foi necessário fazer uma correção topográfica. Quanto ao critério geológico, a direção do perfil foi escolhida de maneira que este cruzasse os lineamentos estruturais. Sendo assim, os perfis geofísicos foram orientados segundo a direção de N45E.

É importante ressaltar que os levantamentos geofísicos foram realizados após um período de cerca de 20 dias de chuvas na região. Como estávamos em busca da localização de zonas de fraturas, esperavase encontrar bons refletores nos perfis GPR, devido ao elevado contraste entre a constante dielétrica da água presente nas fraturas e da rocha encaixante. Além disso, onde o solo de alteração da rocha fosse mais espesso, esperava-se que ele estivesse saturado. Portanto, no caso do GPR esperava-se uma ausência de sinal, ou seja, uma forte atenuação da onda eletromagnética, provocando uma "zona de sombra" no radargrama. Por outro lado, no caso do caminhamento elétrico dipolodipolo esperava-se um aumento na condutividade elétrica, caracterizando uma região anômala que poderia ser facilmente mapeada.

As medidas geofísicas foram realizadas ao longo de um perfil de 80 metros de comprimento. Foram utilizados os métodos: GPR e Eletrorresistividade. Com o método GPR, adquiriu-se um perfil de reflexão com afastamento constante, utilizando-se antenas com freqüências centrais em 50, 100 e 200MHz. Os perfis foram realizados sobre uma mesma linha, visando obter boa penetração e boa resolução, pois os resultados obtidos com as antenas diferentes se complementam. Para as antenas de 50 e 100MHz o espaçamento entre as medidas foi de 0,5m e para as antenas de $200 \mathrm{MHz} 0$ espaçamento foi de $0,25 \mathrm{~m}$. Neste trabalho apresentaremos somente 0 perfil obtido com as antenas de $100 \mathrm{MHz}$, conforme mostra a Figura 2.

Sobre o perfil GPR também foram adquiridas três sondagens de velocidade com as mesmas antenas, utilizando-se a técnica de aquisição WARR - Wide Angle Reflection and Refraction. A análise das sondagens de velocidade permitiu calcular uma velocidade de propagação da onda eletromagnética em subsuperfície de 0,12m/ns, que é compatível com rochas graníticas. Essa velocidade foi utilizada para a conversão de tempo em profundidade.

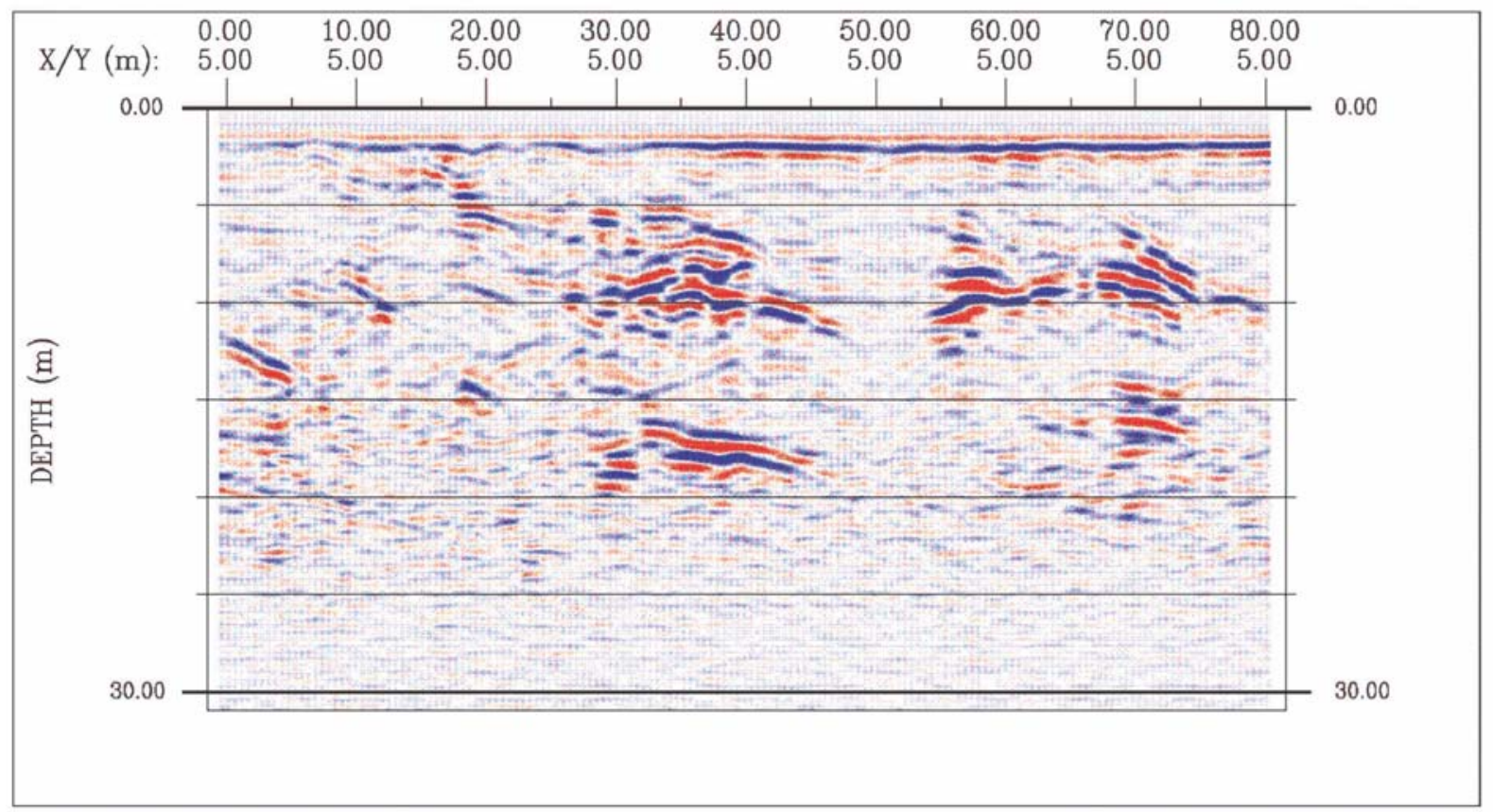

Figura 2 - Perfil de reflexão em profundidade obtido com as antenas de 100 $\mathrm{MHz}$. Figure 2 - Profile of reflection in depth obtained with $100 \mathrm{MHz}$ antennas. 
Os dados GPR foram adquiridos com o equipamento Sueco Ramac (Mala - GeoScience) pertencente ao Departamento de Geofíisica do IAG/ USP. Tanto os perfis de reflexão quanto as sondagens de velocidade foram processados utilizando-se 0 software Gradix (Interpex). 0 processamento básico utilizado consistiu das seguintes etapas: a) aplicação de filtro dc (correção do wow, que é um ruído de baixa frequêencia); b) correção do tempo zero; c) aplicação de ganhos no tempo (filtro do tipo SEC - spherical exponential compensation, linear, constante e programado); d) aplicação de filtro do tipo passa banda; e e) aplicação de filtro espacial moving average (três traços).

Com o método da eletrorresistividade foram adquiridos dois perfis de caminhamento elétrico com arranio dos eletrodos do tipo dipolodipolo: um perfil com espaçamento dos dipolos de $2 \mathrm{~m}$ e 0 outro com espaçamento de 10 metros. 0 perfil de caminhamento elétrico com dipolo de $2 \mathrm{~m}$ é de $50 \mathrm{~m}$ de comprimento. Nesse perfil, fez-se quatro níveis de medidas, 0 que permitiu investigar até cerca de $5 \mathrm{~m}$ de profundidade. 0 perfil de caminhamento com dipolo de $10 \mathrm{~m}$ é de $100 \mathrm{~m}$ de comprimento. Nesse perfil, fez-se cinco níveis de medidas, permitindo investigar as características elétricas da subsuperfície até cerca de $30 \mathrm{~m}$ de profundidade. Ambos os perfis de caminhamento elétrico foram adquiridos sobre os perfis GPR. A Figura 3 mostra o perfil de caminhamento elétrico com dipolos de $10 \mathrm{~m}$.

\section{DISCUSSÃO DOS RESULTADOS}

As análises dos perfis GPR e de caminhamento elétrico dipólodipólo permitiram obter informações da subsuperfície até cerca de 30 metros de profundidade.

Os perfis GPR permitiram obter imagens claras de alguns fortes refletores inclinados ao longo dos perfis. A tendência de mergulho também pode ser observada no perfil de caminhamento elétrico com dipolo de $10 \mathrm{~m}$, através das linhas de isovalores de resistividade elétrica.

Os refletores vistos nos perfis GPR são descontínuos e os dois refletores observados entre 30 e $47 \mathrm{~m}$, um em torno de $10 \mathrm{~m}$ e 0 outro em torno de $17 \mathrm{~m}$ de profundidade, ambos estão interrompidos ao redor da posição de $50 \mathrm{~m}$. 0 primeiro refletor, em torno de 10m de profundidade, é provável que esteja relacionado com a presença de uma fratura inclinada preenchida com água. Esta hipótese é sustentada pela elevada amplitude

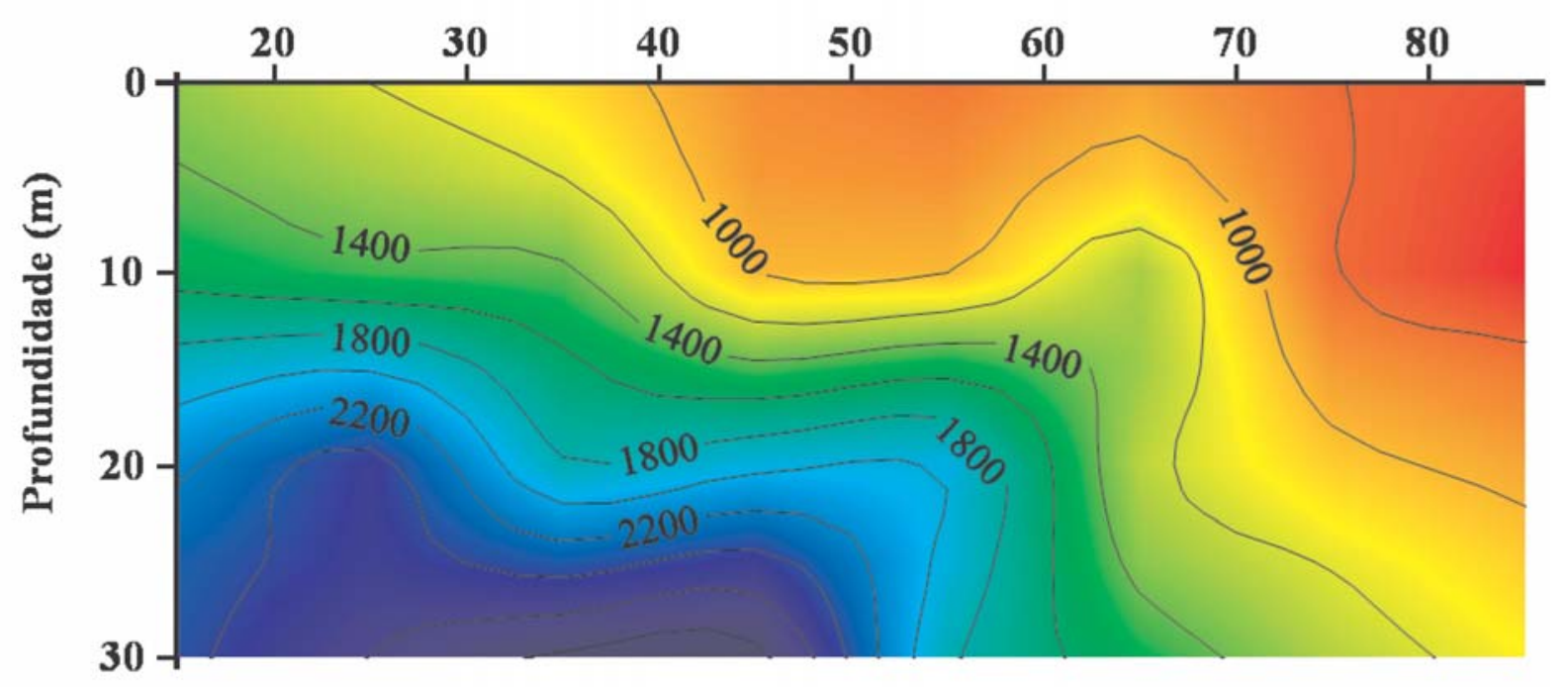

Resistividade Aparente (Ohm.m)

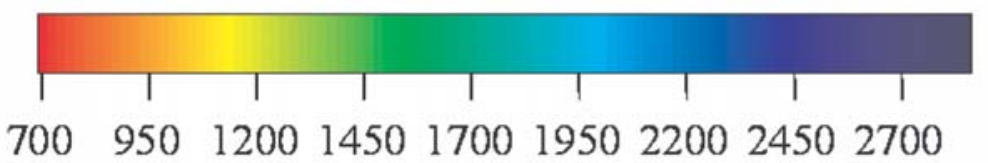

Figura 3 - Perfil de caminhamento elétrico com dipolos de 10m.

Figure 3 - Electric profile obtained by dipole of $10 \mathrm{~m}$. 
do refletor, que é típico de um forte contraste entre a constante dielétrica da água e do granito. Este refletor não tem correspondência com os perfis de caminhamento elétrico dipolar.

Por outro lado, o segundo refletor observado entre as posições de 30 e 47m que está em torno de $17 \mathrm{~m}$ de profundidade, parece ter continuidade para o início do perfil. Note que o nítido refletor que aparece entre as posições de 0 e $5 \mathrm{~m}$ e à profundidade em torno de $12 \mathrm{~m}$ tem a mesma tendência de mergulho e amplitude do refletor anterior, sugerindo ser um único refletor, embora não seja contínuo. É provável que este refletor esteja relacionado com o topo da rocha granítica sã, pois a partir dele não se observam mais refletores, sendo um indicativo da chegada ao topo da rocha. Este refletor tem correspondência com os perfis de caminhamento elétrico dipolar, e pode ser visto através de um aumento na resistividade elétrica nas curvas de isovalores. No início do perfil com dipolo de 10m, ou seja, entre 15 e 55m, observa-se claramente uma região resistiva inclinada a partir dos $15 \mathrm{~m}$ de profundidade, seguindo a mesma tendência de mergulho dos refletores GPR, corroborando com a hipótese de topo da rocha granítica sã.

No perfil de caminhamento com dipolo de 10m, nota-se uma região resistiva com tendência subvertical na posição entre 55 e 65m, ocorrendo desde os $20 \mathrm{~m}$ até cerca de $7 \mathrm{~m}$ de profundidade. Esta feição parece estar relacionada com uma estruturação subvertical na rocha granítica sã. Os trechos com resistividades anômalas são os mesmos e têm correspondência com os perfis GPR, onde se observa maior penetração do sinal eletromagnético, corroborando com a hipótese de rocha granítica menos alterada próxima à superfície.

A região anômala que ocorre nos perfis GPR em torno da posição de $50 \mathrm{~m}$, também aparece no final dos perfis em torno da posição de $80 \mathrm{~m}$. Esta região é caracterizada por uma ausência de reflexão, conhecida como "zona de sombras". Estas regiões também têm correspondência com os perfis de caminhamento elétrico dipolar, sendo caracterizadas por regiões de alta condutividade, facilmente mapeáveis. No perfil com dipolo de $10 \mathrm{~m}$, novamente observam-se duas regiões anômalas condutivas: uma entre 40 e $60 \mathrm{~m}$, e a outra no final do perfil apresentando uma clara indicação de mergulho entre as posições de 70 e $85 \mathrm{~m}$. É provável que estas regiões possam estar relacionadas a uma zona de fraturas subverticais, onde a presença de água acelera o processo de alteração da rocha, aumentando a condutividade elétrica, conseqüentemente, cria-se uma "zona de sombra" nos perfis GPR devido à elevada atenuação do sinal do radar.

\section{CONCLUSÕES}

As investigações geofísicas obtidas com os métodos GPR e eletrorresistividade permitiram imagear a subsuperfície até cerca de 30 metros de profundidade.

Com os pertis GPR foi possível identificar fortes refletores inclinados em torno de $10 \mathrm{~m}$ de profundidade, que são provavelmente fraturas inclinadas preenchidas com água.

Foram identificadas duas regiões anômalas: uma em torno de $50 \mathrm{~m}$ e a outra em torno de $80 \mathrm{~m}$. Nos perfis GPR, estas regiões são caracterizadas por uma "zona de sombra", devido à elevada atenuação das ondas eletromagnéticas. Nos perfis de caminhamento dipolo-dipolo, estas regiões correspondem a regiões condutivas. Estas regiões podem estar relacionadas com a presença de uma zona de fratura subvertical.

No perfil de caminhamento com dipolo de $10 \mathrm{~m}$, nota-se uma região resistiva com tendência subvertical na posição entre 55 e 65m, ocorrendo desde os $20 \mathrm{~m}$ até cerca de $7 \mathrm{~m}$ de profundidade. Esta feição pode estar relacionada com uma estruturação na rocha granítica sã.

A análise dos perfis GPR permitiu identificar claramente um segundo refletor inclinado, que embora descontínuo, aparece no início dos perfis (entre 0 e $5 \mathrm{~m}$ ) à profundidade em torno de $12 \mathrm{~m}$ e entre $30 \mathrm{e}$ $47 \mathrm{~m}$ aparece em torno de $17 \mathrm{~m}$ de profundidade. No perfil de caminhamento com dipolo de $10 \mathrm{~m}$, observa-se uma região resistiva inclinada a partir dos $15 \mathrm{~m}$ de profundidade, seguindo a mesma tendência de mergulho do refletor GPR. É provável que este refletor esteja relacionado com o topo da rocha granítica sã.

\section{REFERÊNCIAS}

ELIS, V. R. Avaliação da aplicabilidade de métodos elétricos de prospecçã̃o geofísica no estudo de áreas utilizadas para disposição de resíduos. 1999. 264 p. Tese (Doutorado)-Instituto de Geociências e Ciências Exatas, Universidade Estadual Paulista, Rio Claro, 1999.

PASCHOLATI, E. M. Caracterização Geofísica da Suíte Intrusiva de Itu. 1990. 135 p. Tese (Doutorado)-Instituto Astronômico e Geofísico, Universidade de São Paulo, São Paulo, 1990.

PORSANI, J. L. Ground Penetrating Rada (GPR): proposta metodológica de emprego em estudos geológico - Goetécnicos nas regiões de Rio Claro e Descalvado - SP. Tese (Doutorado)-Instituto de Geociências e Ciências Exatas, Universidade Estadual Paulista, Rio Claro, 1999. 


\section{NOTAS SOBRE OS AUTORES}

Jorge Luís Porsani é g eólogo pelo Instituto de Geociências da UFBA (1987). Mestrado em Geofísica pelo Núcleo de Pesquisas Geofísicas Aplicadas à Prospecção de Hidrocarnetos da UFPA (1991). De 1991 a 1996, trabalhou como Geofísico no Centro de Pesquisas da PETROBRAS. Doutorado em Geociências e Meio Ambiente pelo Instituto de Geociências e Ciências Exatas da UNESP (1999). Desde 01/12/ 1998 é Docente do Departamento de Geofísica do IAG/USP, atuando com métodos geoelétricos aplicados à geologia, geotecnia, meio ambiente e arqueologia. Atualmente, é vice-coordenador do Programa de Pós-Graduação em Geofísica e coordenador do Projeto de Instalação do Sítio Controlado de Geofísica Rasa do IAG (Fapesp 02/07509-1).

Francisco Yukio Hiodo é graduado em Física pelo Instituto de Física da Universidade de São Paulo-USP em 1972. Mestrado em Geofísica pelo Instituto de Astronomia, Geofísica e Ciências Atmostéricas (IAG-USP) em 1981. Doutorado em Geofísica pelo IAG-USP em 1990. Auxiliar de Ensino no IAG-USP em 1973. Professor Assistente em 1981. Professor Doutor do IAG-USP desde 1990. Atualmente é especialista em Instrumentação Geofísica nas áreas de Paleomagnetísmo (magnetômetros rotativos e desmagnetizadores), Geofísica Nuclear (espectrômetros gama e emanômetros de radônio), Perfilagem de poços tubulares, Geotermometria e sensores de temperatura, Magnetômetros de precessão de prótons e fluxgate, Métodos geoelétricos e eletromagnéticos.

Vagner Roberto Elis é graduado em Geologia em 1990 pela Universidade Estadual Paulista. Mestre em Geociências pela Universidade Estadual Paulista em 1993, desenvolvendo a dissertação "A aplicação da geofísica para análise do meio físico: Importância para elaboração de mapeamento geotécnico". Doutor em Geociências pela Universidade Estadual Paulista em 1999, defendendo a tese "Avaliação da aplicabilidade de métodos elétricos de prospecção geofíisica no estudo de áreas utilizadas para disposição de resíduos". Docente do Departamento de Geofísica do Instituto de Astronomia, Geofísica e Ciências Atmosféricas da Universidade de São Paulo, com atuação nas áreas de Geofísica Aplicada a estudos ambientais e hidrogeológicos. 